\title{
Universitäre Forschung in Naturheilkunde und Komplementärmedizin - Perspektiven und Standortbestimmung
}

Der Blick auf das menschliche Leben und auf Gesundheit und Krankheit ist in hohem Masse abhängig von der Perspektive und den Methoden der Erkenntnisgewinnung der jeweiligen Wissenschaften. Im Kontext moderner Wissenschaftstheorien existieren klassische Grundmuster von Wirklichkeitskonstruktionen der Wissenschaften, die als biomedizinische, existentiell-anthropologische, kulturologische und systemische Paradigmen bezeichnet werden können [1]

Diese unterschiedlichen wissenschaftstheoretischen Perspektiven gelten für alle wissenschaftlichen Fragestellungen - auch für eine Forschung in der Naturheilkunde und Komplementärmedizin. Biomedizinische und anthropologische Paradigmen fokussieren mit ihren Fragestellungen vermehrt auf die Person und Biographie sowie den Organismus des Menschen [2]. Hier sind u.a. Physiologie und Verhaltensweisen primäre Forschungsgegenstände. Systemische und kulturologische Paradigmen beziehen verstärkt die Verhältnisweisen in ihre Betrachtungen mit ein und können als ökologische und ethnographische Modelle bezeichnet werden [2]. Nur alle Perspektiven zusammen erlauben umfassende Beurteilungen von grundlegenden Lebensprozessen, deren Realität immer von komplexen Beziehungen zwischen Umwelt und Person abhängig ist.

Die Medizin wird vorwiegend im biomedizinischen Paradigma der Naturwissenschaften betrachtet. Die Szientistik mit ihrem naturwissenschaftlichen Methodenspiegel unterscheidet die Nachweiskategorien von Wirkung (Effekt), Wirkprinzip und Wirksamkeit.

Die Wirkungsnachweise werden an unterschiedlichen Modellen und deren Parametern durchgeführt (z.B. Beeinflussung bestimmter Immunparameter der Maus oder des Menschen) und können in ihrer Gesamtheit mögliche objektive Aufschlüsse über Wirkprinzipien von medizinischen Behandlungsmethoden geben, die für das - vorwiegend naturwissenschaftliche - Verständnis hinsichtlich ihrer Plausibilität von besonderer Bedeutung sind.

Die traditionelle klinische Forschung versucht primär die Wirksamkeit von Interventionen mit Hilfe kontrollierter klinischer Studien zu untersuchen. Hier steht meist das einzelne
Diagnose- bzw. Behandlungsverfahren, d.h. die Intervention als solche, im Vordergrund der Untersuchung. Es wird der spezifische Effekt der Massnahme und die Ursächlichkeit des Zusammenhanges zwischen Intervention und Ergebnis sowie die Generalisierbarkeit und Beweisbarkeit erforscht. Ein wissenschaftlicher Wirksamkeitsnachweis im engeren Sinne ist jedoch für die in der Naturheilkunde und Komplementärmedizin sehr unterschiedlichen und im zeitlichen Verlauf variablen «Therapiecocktails» oft schwer zu erbringen. Meist fehlt die für eine Verallgemeinerung notwendige Repräsentativität und Reproduzierbarkeit der Methode. Pflanzenextrakte enthalten häufig keine standardisierten Inhalte, Akupunkturbehandlungen werden in Form verschiedenster Techniken, in Anlehnung an unterschiedliche Schulmeinungen und abhängig von der persönlichen Therapeutenqualifikation ausgeübt; Anwendungen werden - je nach Behandler und Schulmeinung - in Ergänzung oder als Alternative zur konventionellen Therapie durchgeführt. Viele offene Fragen, die es im Vorfeld von experimentellen Studien hinsichtlich der Repräsentativität und klinischen Relevanz im Konsens der Fachexperten zu beantworten gilt. Die Prüfung einzelner Bausteine in randomisierten Studien wäre wünschenswert, ist aber bei den meist sehr individualisierten und klinikspezifischen Vorgehensweisen oft nur von begrenzter Aussagefähigkeit hinsichtlich ihrer Ergebnis-Verallgemeinerung.

Als eine idealtypische Studienform bei der Feststellung der klinischen Wirksamkeit gilt die randomisierte, plazebokontrollierte klinische Studie. An diesem speziellen Beispiel einer klinischen Forschung sollen folgende Ausführungen gemacht werden.

Formal lässt sich folgende Modellstruktur darstellen [3]:

$$
\begin{aligned}
& \text { TOT }=\text { NC }+ \text { PL }+ \text { SP }+ \text { BIAS }+ \text { CHANCE } \\
& \frac{\text { TOT }=\text { NC }+ \text { PL }+\ldots .+ \text { BIAS }+ \text { CHANCE } . /}{\text { Specific effect }}
\end{aligned}
$$

Der Totaleffekt (TOT) einer Behandlungsmassnahme setzt sich aus verschiedenen Teilkomponenten zusammen. Zunächst ist dies der natürliche Verlauf $(N C)$ einer Erkrankung,

\begin{tabular}{ll}
\hline KARGER & @ 2003 S. Karger GmbH, Freiburg \\
$\begin{array}{l}\text { Fax +497614520714 } \\
\begin{array}{l}\text { E-mail Information@Karger.de } \\
\text { www.karger.com }\end{array}\end{array}$ & $\begin{array}{l}\text { Accessible online at: } \\
\text { www.karger.com/fkm }\end{array}$
\end{tabular}


ihre patho-physiologische Eigenbestimmung, aber auch die salutogenen Schutzfaktoren, die autoregulativen Kompetenzen oder Ressourcen eines Organismus bzw. eines Patienten/Klienten. Diese autonomen, organismuseigenen und biographisch-persönlichkeitsabhängigen Fähigkeiten und Fertigkeiten des Menschen zur Selbstheilung, Selbstkompetenz und Reifung sind Hauptziel der meisten therapeutischen Bemühungen von komplementärmedizinischen Verfahren. Im Modell der Selbstheilung findet sich bei den sonst so unterschiedlichen Verfahren der Komplementärmedizin eine gemeinsame Theorienbildung. Diese wichtige Teilkomponente und ihre therapeutische Beeinflussung durch «Naturheil-verfahren» wird jedoch bislang kaum untersucht.

Eine weitere wichtige Komponente für die Bildung des Gesamteffektes einer Behandlung ist der Plazeboeffekt $(P L)$, der selbst zunehmend Gegenstand von wissenschaftlichen Untersuchungen wird. Hier kann mit Recht ein weiterer wesentlicher Anteil der Wirkung von komplementärmedizinischen Interventionen vermutet werden. «Plazebo» hat mit «mir gefällt etwas» zu tun und spricht damit den individuellen Bereich der Glaubens- und Wertemuster von Individuen an. Hieraus leiten sich Glaubenssätze der Akteure (Patienten wie Behandler!) ab, die auch die Handlungs- und somit Behandlungsmuster massgeblich beeinflussen. Die subjektiven Lebens- und Krankheitstheorien der Patienten und Ärzte stimmen deshalb auch oft - zumindest in wesentlichen Teilen überein und bilden die Grundlage für eine Therapeuten-Patienten-Interaktion und einer gemeinsamen Diagnose- und Behandlungsrealität. In diesem Zusammenhang bilden Farbe und Geschmack eines Medikamentes, der weisse Kittel des Therapeuten, der unbekannte und geheimnisvolle Wirkungsmechanismus einer Intervention sowie die Form und das Aussehen einer instrumentellen Methode eine ganz individuelle Beziehungs- und Bedeutungsdefinition, die mit einer rationalen inhaltlichen Definition und möglichen wissenschaftlichen Wirkung nichts mehr gemein hat. Der Plazeboeffekt umschreibt in diesem Sinne auch einen eigenständigen «Faktor von Versorgungsqualität» bzw. einen «CARE-Faktor», der unter anderem von der Persönlichkeit und dem Setting des medizinischen Dienstleisters mit beeinflusst wird. Diese Plazebowirkung ist damit meist untrennbar mit der interaktionsintensiven Beziehung zwischen Patient und Therapeut verbunden und kann nur unter Experimentalsituationen aus dieser funktionellen und personalen Einheit genommen werden. Weitere plazebobezogene Kontextfaktoren können auch wirtschaftliche Anreize, z.B. kostenlose Behandlung oder Refinanzierungsmöglichkeiten, sowie situationsbedingte Einflüsse wie Stimmungen und emotionale Vorfälle sein.

Die Evaluation klinischer plazebokontrollierter Studien bezieht sich jedoch bislang vorwiegend auf die Untersuchung eines spezifischen Effekts (SP), einer z.B. pharmakologischen Wirkung oder Wirksamkeit einer Intervention, indem diese bislang erwähnten Einflussgrössen einschliesslich «Bias» (Selbsttäuschung) und Zufall (Chance) durch den Prozess der
Randomisation auf zwei zu untersuchende Patientenkollektive durch Zufall gleichmässig verteilt werden - ausser dem zu untersuchenden spezifischen Effekt (SP) der Intervention selbst (TOT-TOT ohne «specific effect»). Nur unter dieser experimentellen Bedingung lassen sich - aus biometrischer Sicht - die besten Aussagen über den kausalen Zusammenhang zwischen einem beobachtbaren therapeutischen Ergebnis und einer Intervention treffen.

Im Gegensatz zu kontrollierten klinischen Studien wird mit dem Ansatz der patientenzentrierten Ergebnis-Qualitätsbewertung nicht die Wirksamkeit einer Intervention geprüft, sondern die Anwendung und Auswirkung von Interventionen in der klinischen Routine bzw. im ärztlichen Alltag abgebildet und die «real world effectiveness» (alltagsnaher Nutzen) dargestellt und bestimmt.

Eine in diesen Bereich gehörende Studienform ist die «Patient Care Evaluation Study» (PCES), die in der medizinischen Wissenschaft - bevorzugt im angloamerikanischen Sprachraum - für die Überprüfung der Umsetzung von Standards und Leitlinien in die medizinische Versorgungswirklichkeit und deren tatsächliche Auswirkung auf die Patientenversorgung benutzt wird [4].

Aufgrund der fehlenden Wissenschaftstradition und der unterschiedlichen Entwicklungen der einzelnen komplementär-medizinischen Richtungen kann jedoch im vorliegenden Falle nicht die Überprüfung der Einhaltung von Evidenz-basierten Leitlinien oder die Umsetzung der Erkenntnisse aus klinischen Studien Ziel des Vorhabens sein. Vielmehr geht es bei der Durchführung von derartigen Beobachtungsstudien um Transparenz und Darstellung von Diagnostik, Therapie und Verlauf von Patientenbehandlungen sowie um die Evaluation von deren Auswirkungen auf z.B. den selbstempfundenen Patientennutzen, die Patientenzufriedenheit, die Sicherheit, Verträglichkeit und das Kosten-Nutzen-Verhältnis sowie den klinischen Nutzen. Hierfür ist ein alltagsnahes und arbeitsplatzbezogenes Dokumentationssystem zu entwickeln, das auswertbare und valide Daten für ein multizentrisches Register liefert. Dies erlaubt Quer- und Längsschnittauswertungen mit (zugegeben nicht problemfreien) Vergleichen.

Die Versorgungsqualität spielt somit - neben der Wirksamkeit - eine zunehmende Rolle in der Steuerung der Medizin. Dies ist der Grund dafür, dass die Qualitäts- und Versorgungsforschung auch einen zunehmenden Stellenwert in der universitären klinischen Forschung erfährt.

Das Qualitätsmanagement verfolgt das Ziel, die «Wie-Beschaffenheit» (Qualität) von Strukturen und Prozessen festzustellen und insbesondere Therapieergebnisse eines bestimmten Anbieters kontinuierlich und systematisch unter Praxisbedingungen zu dokumentieren und zu verbessern. Hier stehen die Beurteilung der gesamten Betreuungssituation des einzelnen Anbieters und der globale Nutzen (TOT) für den einzelnen Patienten im Vordergrund der Betrachtung. Dieser Ansatz beschränkt sich meist nicht auf das medizinisch definierbare Ergebnis, sondern bezieht gesundheitsorientierte, pro- 
zessbezogene, wirtschaftliche und allgemeine (generische) Resultate der Interventionen, wie z.B. Beeinflussung von Risikofaktoren, allgemeinen Gesundheitszustand, Lebensqualität und Patientenzufriedenheit mit ein. Die kontinuierliche und patientenzentrierte Qualitätsbewertung einzelner Leistungserbringer ist aber eine wichtige Ergänzung zu den Evidenzdaten aus kontrollierten klinischen Studien. Das Konzept einer «evidence-based medicine» (EBM) erfährt somit eine sinnvolle Erweiterung in Richtung einer «confidence-based medicine» $(C B M)$, die den einzelnen Anbieter durch Qualitätsdarstellung noch vertrauenswürdiger macht [5]. Erst gemeinsam bilden sie für Patienten und zuweisende Ärzte die rationale Grundlage einer individuellen Inanspruchnahme-Entscheidung.

D. Melchart, München

\section{Literatur}

1 Lafaille R: Auf dem Weg zu einer Gründung der Gesundheitswissenschaften Möglichkeiten, Herausforderungen, Fallstricke; in Göpel E, Schneider-Wohlfahrt U (Hrsg.): Provokationen zur Gesundheit. Beiträge zu einem reflexiven Verständnis von Gesundheit und Krankheit. Frankfurt, Mabuse, 1994, pp 229-266.

2 Göpel E: Multimedialer Kooperationsverbund Hochschulen für Gesundheit. Sommerakademie zur Gesundheitsförderung. Magdeburg, 2001.

3 Kleijnen J: Placebos and placebo effects in clinical trials. State-of-the-Science Symposia. Harvard Medical School. Syllabus 1999;402-403.

4 Hölzer S, Dudeck J: Patient Care Evaluation Studies - ein umfassendes Konzept zur Evaluation der onkologischen Patientenversorgung. ZaeFQ 2000;94:121-126.

5 Melchart D: Kontinuierliche Integration von Qualitätsmanagment in die ärztliche Praxis; in Melchart, Brenke, Dobos, Gaisbauer, Saller (Hrsg.): Naturheilverfahren 2002, pp 616-632. 